\title{
Sex differences and predictors of objective cough frequency in chronic cough
}

\author{
A Kelsall, ${ }^{1,2}$ S Decalmer, ${ }^{1}$ K McGuinness, ${ }^{1,2}$ A Woodcock, ${ }^{1,2} \mathrm{~J} \mathrm{~A} \mathrm{Smith}^{1}$
}

\section{See Editorial, p 372}

- Additional figures are published online only at http:// thorax.bmj.com/content/vol64/ issue5

${ }^{1}$ Respiratory Research Group, University of Manchester, University Hospital of South Manchester NHS Foundation

Trust, Manchester, UK; ${ }^{2}$ North

West Lung Centre, University Hospital of South Manchester NHS Foundation Trust,

Manchester, UK

Correspondence to: Dr J A Smith, Education and Research Centre, Wythenshawe Hospital, Southmoor Road, Manchester M23 9LT, UK; jacky.smith@manchester.ac.uk

Received 26 August 2008 Accepted 5 December 2008 Published Online First

6 January 2009

\begin{abstract}
Background: Women are consistently over-represented in specialist cough clinics and known to have a more sensitive cough reflex than men. Whether female sex and other patient characteristics are associated with higher cough rates is not known. A study was conducted to determine the predictors of objective cough frequency in patients presenting to a tertiary referral clinic with chronic cough.
\end{abstract}

Methods: 100 subjects (65 women) of mean (SD) age $55.8(11.0)$ years and median cough duration 4 years (IOR 2.0-10.0) with unexplained chronic cough completed flow-volume loops (mean (SD) forced expiratory volume in 1 s 103 (15.2)\% predicted; forced expiratory flow ( $\left.\mathrm{FEF}_{50}\right)$ 68.8 (24.1)\% predicted), methacholine challenge (42\% positive), citric acid cough reflex sensitivity (C5; $0.12 \mathrm{M}$ (IQR 0.06-0.50)) and the Leicester Cough Questionnaire. 24-h ambulatory cough monitoring was performed in 86 subjects; manually counted coughs were quantified as the number of explosive cough sounds per hour.

Results: Women coughed significantly more than men (geometric mean 16.6 coughs/h (95\% Cl 13.1 to 21.0 ) vs 9.4 coughs/h (95\% $\mathrm{Cl} 6.4$ to 13.9), $p=0.01)$ ). The cough reflex was also more sensitive in women than in men (median logC5 $-0.9 \mathrm{M}$ vs $-0.6 \mathrm{M}, \mathrm{p}=0.002$ ), but cough-related quality of life was similar in women and men (12.0 (3.6) and 12.2 (3.2), respectively, $p=0.76$ ). Linear regression analysis showed that $38.6 \%$ of the variation in cough rate was predicted by sex $(p=0.01)$, $\log C 5(p<0.001)$ and age $(p=0.002)$ but not lung function or bronchial hyper-reactivity.

Conclusions: Ambulatory objective cough monitoring provides novel insights into factors modulating chronic cough. These findings suggest that effects of sex and age must be taken into account in the study of cough and when designing clinical trials testing novel antitussive agents.

Cough is a common and troublesome symptom with chronic cough estimated to affect $14-23 \%$ of the adult population. ${ }^{1}$ There is evidence that the prevalence of chronic cough may be greater in adult women than men. For example, a large international population survey found that reporting of nocturnal and non-productive cough was related to female sex, ${ }^{2}$ and a UK survey suggested daily coughing was more commonly reported by women than men. ${ }^{3}$ Furthermore, in specialist clinics caring for patients with chronic cough, female patients are consistently over-represented, ${ }^{4-11}$ particularly among those with idiopathic cough. ${ }^{12}$ This apparent female preponderance may be due to a true increased prevalence of chronic cough, but it has been speculated that behavioural differences could account for the discrepancy with women being more likely to access health care. Women may be driven to seek medical advice by more troublesome complications of coughing such as stress urinary incontinence $^{13}$ and the associated impact on quality of life. ${ }^{13} 14$

In addition to increased reporting of cough, the cough reflex is more sensitive to tussive agents in women. This effect is seen in both healthy subjects ${ }^{15-18}$ and those with chronic cough, ${ }^{19}$ but the underlying mechanisms are not understood. More importantly, there are no data assessing whether women experience more severe coughing then men (ie, higher objective cough frequencies) either in health or in those suffering from chronic cough.

We tested the hypothesis that women with chronic cough have greater cough frequencies than men. We have studied patients with chronic cough attending a specialist cough clinic to identify and quantify factors including sex which predict objective cough frequency determined by 24-hour ambulatory cough monitoring.

\section{METHODS \\ Subjects}

Consecutive patients complaining of chronic cough (>8 weeks duration) attending a tertiary specialist cough clinic (North West Lung Centre, University Hospital of South Manchester, UK) were studied. Current and ex-smokers of $<6$ months, patients taking angiotensin converting enzyme inhibitors and opiates, and patients with significant comorbidities (eg, diabetes, heart failure, chronic obstructive pulmonary disease) were excluded. The following tests were performed.

\section{Cough assessments}

\section{Ambulatory cough monitoring}

Twenty-four hour ambulatory cough sound recordings were performed using a custom-built recording device with an air microphone and chest wall sensor (Vitalojak, Vitalograph, UK). Recordings were manually counted by a single trained observer and expressed as the number of explosive cough sounds per hour. ${ }^{20}$ Subjects were encouraged to continue their normal routine throughout the recording period and noted the time of going to bed.

\section{Cough reflex sensitivity}

Citric acid challenge was used to assess cough reflex sensitivity. ${ }^{21}$ Doubling doses (from $0.03 \mathrm{M}$ to 4.0 M citric acid, Stockport Pharmaceuticals, UK) were administered as single breath inhalations (KoKo dosimeter, Pds Instrumentation, USA) with three placebo inhalations of normal saline 
randomly interspersed in a double-blind manner. Following each inhalation, the number of coughs in the subsequent minute was counted by an experienced observer and recorded. The challenge was terminated once the citric acid induced 5 or more coughs and the concentrations provoking 2 and 5 coughs were recorded (C2 and $\mathrm{C} 5)$.

\section{Leicester Cough Questionnaire (LCQ)}

Cough-related quality of life was measured using the LCQ, a validated questionnaire comprising 19 questions in three domains (physical, psychological and social). ${ }^{22}$ Possible total scores ranged from 3 to 21 and domain scores from 1 to 7 , with higher scores representing better quality of life.

\section{Pulmonary function tests}

\section{Flow-volume loops}

Three technically acceptable and reproducible forced expiratory flow-volume loop manoeuvres were performed to ATS/ERS standards $^{23}$ (Jaeger Masterscreen, Erich Jaeger, Germany). The highest values recorded were used for analysis.

\section{Bronchial hyper-reactivity (BHR) testing}

BHR testing was performed using methacholine chloride solutions (Stockport Pharmaceuticals, UK) and the modified Yan method. ${ }^{21}$ Doubling doses (to a maximum of $5.96 \mathrm{mg} / \mathrm{ml}$ ) were delivered by dosimeter (KoKo dosimeter) in order to achieve a provocative dose $\left(\mathrm{PD}_{20}\right)$ expressed as a $20 \%$ decrease in forced expiratory volume in $1 \mathrm{~s}\left(\mathrm{FEV}_{1}\right)$ from baseline.

\section{Diagnosis}

Final diagnoses were achieved based on a combination of investigations and treatment trials as recommended by the BTS guidelines. $^{24}$

\section{Statistical analysis}

Analyses were performed using SPSS Version 13.0 (SPSS Inc, Chicago, Illinois, USA) and the standard 5\% level was used for statistical significance throughout. Cough rates and cough reflex sensitivity data (C5) were log transformed to allow for parametric analysis where appropriate. Comparisons between male and female subjects were performed using unpaired $t$ test or the Mann-Whitney $U$ test. Relationships between objective cough frequency and continuous variables were assessed by correlation. To explore whether sex differences in objective cough frequency were a consequence of sex differences in other parameters (eg, cough reflex sensitivity), linear regression analyses were performed. Variables found to significantly influence cough frequency in the univariate analyses were included as the independent variables, and all models were adjusted for any effect of age.

Based on previous data in patients with chronic cough ${ }^{25}$ with a geometric mean 24-hour cough frequency of 12.9 coughs/h (95\% CI 10.1 to 16.1), a sample size of 100 subjects (assuming ratio of men to women of $1: 2$ ) would have $90 \%$ power to detect a $15 \%$ difference in cough frequency between sexes.

\section{RESULTS \\ Subjects}

One hundred subjects (65 women) of mean (SD) age 55.8 (11.0) years and median cough duration 4.0 years (IOR 2.010.0) with chronic cough were studied and, of these, 86 subjects agreed to and completed 24-hour cough monitoring. A comparison of the demographic characteristics and pulmonary physiology for male and female patients is shown in table 1. Male and female subjects had similar characteristics. All subjects achieved satisfactory $\mathrm{FEV}_{1}$ measurements, but two subjects were unable to complete full flow-volume loops due to excess coughing. There was evidence of reduced $\mathrm{FEF}_{50}$ values in both women and men, which may represent increased airflow resistance in smaller airways. Positive methacholine challenges were found in 38 patients (44.2\%) and 23 (26.7\%) had a previous diagnosis of asthma.

Following collection of these data, subjects underwent further investigations and treatment trials to establish the underlying causes of their cough. A diagnosis was assigned if they reported a partial or good response to specific treatment; diagnoses were gastro-oesophageal reflux disease $(n=45)$, rhinosinusitis $(\mathrm{n}=27)$, asthma $(\mathrm{n}=16)$, eosinophilic bronchitis $(n=6)$, bronchiectasis $(n=6)$ and idiopathic $(n=25)$. Note that some subjects had more than one diagnosis, and in 25 subjects either the cough was resistant to treatment of identified conditions or no cause could be found.

\section{Ambulatory cough monitoring}

The geometric mean 24-hour cough rate was 13.9 coughs/h (95\% CI 11.3 to 17.0 ). Women coughed significantly more than men (fig 1). Cough frequency in women was not significantly higher during the daytime (at the $5 \%$ level, $p=0.06$ ), but was significantly higher during the night compared with men (fig 2 , table 2).

Patients with idiopathic cough (15 women (60\%), 10 men $(40 \%))$ had similar objective cough frequencies to patients with "explained" cough (24-hour cough frequency, $p=0.75$; day, $\mathrm{p}=0.90$; night, $\mathrm{p}=0.57$ ).

\section{Cough reflex sensitivity}

Two subjects coughed on inhalation of normal saline (placebo) so $\mathrm{C} 5$ could not be measured. For the remaining 98 patients, the median C5 was $0.12 \mathrm{M}$ (IOR 0.06-0.50) and C2 was $0.06 \mathrm{M}$ (IOR 0.03-0.06). Women had a significantly more sensitive cough reflex than men for $\mathrm{C} 5$, a difference of 1 doubling dose

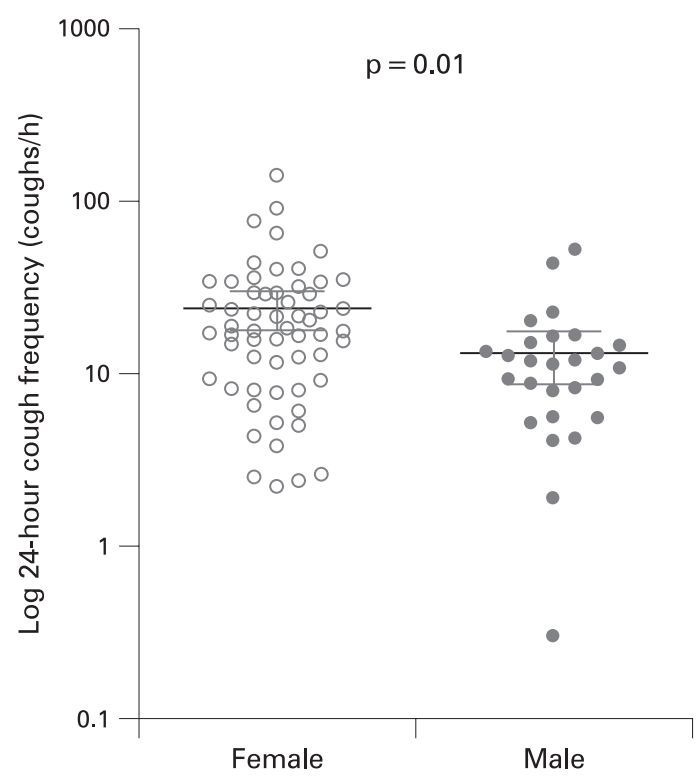

Figure 1 Effect of sex on 24-hour cough frequency. Note logarithmic scale (mean and 95\% confidence interval shown). 
Table 1 Characteristics of men and women with chronic cough undergoing ambulatory cough recording

\begin{tabular}{lccc}
\hline & $\begin{array}{c}\text { Women } \\
(\mathbf{n}=\mathbf{5 9})\end{array}$ & $\begin{array}{l}\text { Men } \\
(\mathbf{n}=\mathbf{2 7})\end{array}$ & p Value \\
\hline Age (years) & $55.3(10.9)$ & $58.3(9.4)$ & 0.23 \\
$\mathrm{BMI}\left(\mathrm{kg} / \mathrm{m}^{2}\right)$ & $28.5(5.2)$ & $27.0(2.7)$ & 0.08 \\
Reported cough history (years) & $3(\mathrm{IQR} 2-10)$ & $3(\mathrm{IQR} 1.5-10)$ & $0.68 \dagger$ \\
$\mathrm{FEV}$ & $(\%$ predicted) & $103.4(16.5)$ & 0.99 \\
$\mathrm{FEV}_{1} / \mathrm{FVC}$ ratio & $103.3(15.3)$ & $74.1(6.9)$ & 0.11 \\
$\mathrm{FEF}_{50}(\%$ predicted) & $76.9(7.8)$ & $71.6(24.6)$ & 0.40 \\
Bronchial hyper-reactivity $_{\left(\mathrm{PD}_{20}<5.96 \mathrm{mg} / \mathrm{dl}\right)}$ & $66.9(23.4)$ & $9 / 27(33 \%)$ & $0.17^{*}$
\end{tabular}

Comparisons made by independent $t$ test except for those marked ${ }^{*} \chi^{2}$ test and $†$ Mann-Whitney test.

$\mathrm{BMI}$, body mass index; $\mathrm{FEF}_{50}$, forced expiratory flow; $\mathrm{FEV}_{1}$, forced expiratory volume in $1 \mathrm{~s} ; \mathrm{PD}_{20}$, dose provoking a fall in $\mathrm{FEV}_{1}$ of $20 \%$ or more.

(fig 3, table 2). For the $\mathrm{C} 2$ endpoint, the difference did not reach significance at the $5 \%$ level.

\section{Leicester Cough Questionnaire (LCO)}

The total LCQ score was 12.1 (3.4) and there were no significant differences between cough-related quality of life for women and men (see fig 5 in online supplement). Of the three domains of the scale, none showed a significant sex difference (see fig 6 in online supplement). However, the $\mathrm{p}$ value for the physical domain was low, suggesting a possible effect with men tending to score more highly (better quality of life) than women.

\section{Predictors of cough frequency}

Cough rate negatively correlated with cough reflex sensitivity $(\log C 5, r=-0.47, p<0.001 ; \log C 2, r=-0.23, p=0.05$; fig 4). There was a weak positive correlation between age and cough frequency $(r=0.20, p=0.07)$ which did not quite reach statistical significance (see fig 7 in online supplement). However, cough frequency was not related to the reported cough history $(r=-0.03, p=0.78)$, lung function parameters $\left(F_{1}: r=0.02, p=0.83 ; F_{5 F}: r=-0.12, p=0.29\right)$ or the level of $\mathrm{BHR}\left(\mathrm{PD}_{20}\right.$ methacholine: $\left.\mathrm{r}=-0.22, \mathrm{p}=0.19\right)$. Subjects with a positive rather than negative methacholine challenge did not cough more by day (median 18.4 (IOR 9.3-33.6) vs median 20.9 (IOR 12.7-34.5), $\mathrm{p}=0.49$ ) or by night (median 4.4 (IOR 1.910.4) vs 2.7 (IOR 0.3-7.6), $p=0.18$ ) and ex-smokers did not cough more than non-smokers $(p=0.54)$.

The interactions between predictors of objective cough frequency were assessed for 24-hour and night cough frequency as significant sex differences were found for these parameters. Cough frequency (24-hour) was independently predicted by sex $(p=0.01)$, age $((B=0.01(95 \%$ CI 0.004 to 0.018$), p=0.002)$ and cough reflex sensitivity $\log \mathrm{C} 5(\mathrm{~B}=-0.38(95 \% \mathrm{CI}-0.52$ to $-0.24), p<0.001)$. These variables accounted for $38.6 \%$ of the variance in 24-hour cough frequency. Figure 4 shows how the relationships between cough reflex sensitivity and cough frequency differ between men and women. In subjects with the most sensitive cough reflexes, men and women had similar cough frequencies during the 24-hour monitoring period but, in those subjects with least sensitive cough reflexes, women coughed significantly more than men (see fig 4A, note cough frequency is plotted on a logarithmic scale).

Night cough was statistically significantly predicted by sex $(p<0.001)$, age $(B=0.014$ (95\% CI 0.007 to 0.022$), p=0.004)$ and $\log \mathrm{C} 5(\mathrm{~B}=-0.22(95 \% \mathrm{CI}-0.37$ to -0.07$), \mathrm{p}<0.001)$, explaining $36.4 \%$ of the variance. Overnight the slopes of the
Figure 2 Day and night objective cough rates in women and men (median and interquartile ranges).
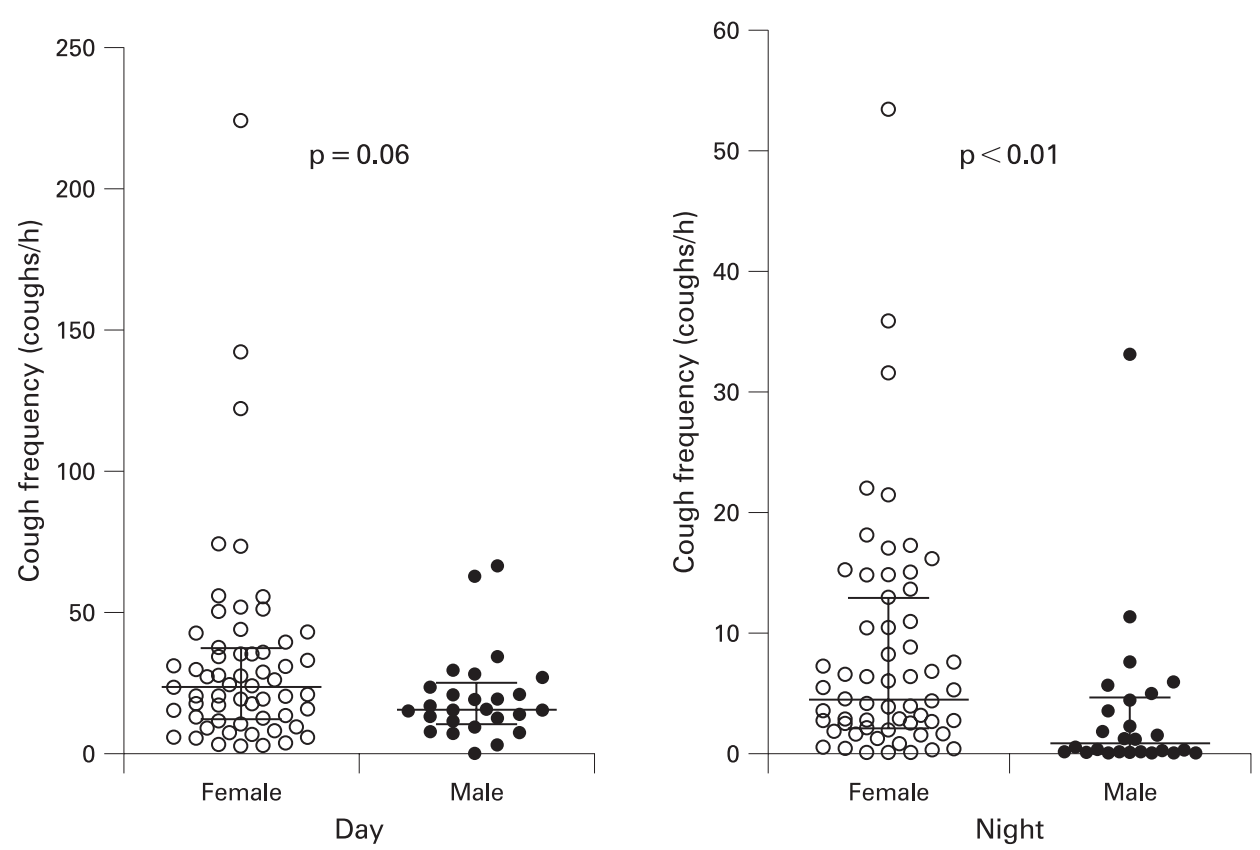


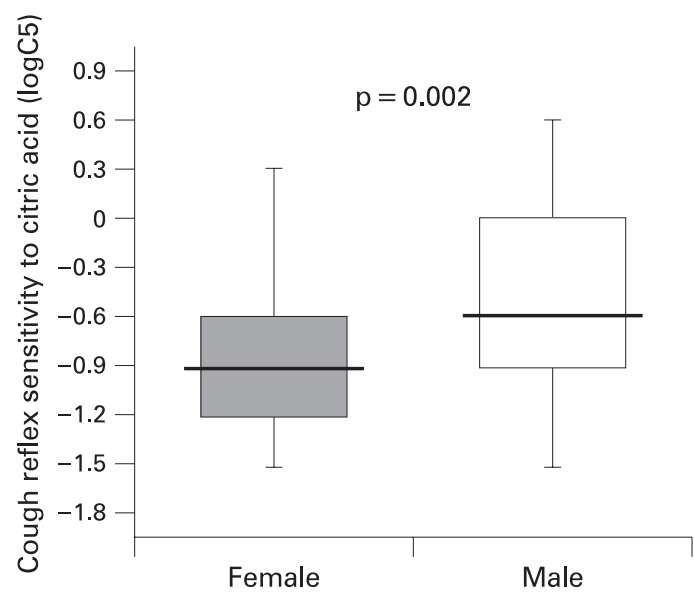

Figure 3 Box plot showing the effect of sex on cough reflex sensitivity (C5). Error bars indicate range, median and 25th and 75th percentiles.

relationships between cough reflex sensitivity and cough frequency were very similar but, for women, the relationship was shifted towards greater cough frequencies for subjects with similar cough reflex sensitivities, regardless of the level (fig 4B).

\section{DISCUSSION}

This study shows for the first time that women presenting to a specialist cough clinic have significantly higher objective cough rates than men, particularly overnight. This observation, along with the effect of age on cough frequency, needs to be taken into account when studying cough frequency and designing clinical trials testing antitussive drugs. The greater cough rate experienced by women with chronic cough may explain their over-representation in specialist clinics, in addition to differences in behaviour or the impact of coughing on quality of life although in this study-using the LCQ - there were no sex differences in cough-related quality of life.

The mechanisms underlying these and previous sex differences observed in chronic cough are uncertain. Consistent with previous reports, ${ }^{16}{ }^{19}$ we have found cough reflex sensitivity to citric acid was heightened in women with chronic cough and moderately correlated with cough counts. ${ }^{25}$ Observational studies have suggested that sex hormones may modulate cough reflex sensitivity. The heightened cough reflex sensitivity in women compared with $\operatorname{men}^{15}{ }^{17}$ has not been found in

Table 2 Measures of cough in women and men

\begin{tabular}{|c|c|c|c|}
\hline & Women & Men & p Value \\
\hline \multicolumn{4}{|c|}{ Cough frequency, coughs $/ \mathrm{h}(\mathrm{n}=86$ ) } \\
\hline 24-hour* & 16.6 (13.1 to 21.0$)$ & $9.4(6.4$ to 13.9$)$ & 0.01 \\
\hline Day† & $23.7(12.4-37.5)$ & $15.6(11.1-24.3)$ & 0.06 \\
\hline Night $\uparrow$ & $4.5(2.1-12.9)$ & $0.8(0.1-4.5)$ & $<0.001$ \\
\hline \multicolumn{4}{|c|}{ Cough reflex sensitivity, $M(n=98)$} \\
\hline $\log _{10} \mathrm{C} 5 \dagger$ & $-0.9(-1.2$ to -0.6$)$ & $-0.6(-0.9$ to 0.0$)$ & 0.002 \\
\hline $\log _{10} \mathrm{C} 2 \uparrow$ & $-1.12(-1.5$ to -0.76$)$ & $-0.9(-1.22$ to -0.6$)$ & 0.06 \\
\hline \multicolumn{4}{|l|}{ LCO $(n=100)$} \\
\hline Total: & $12.0(3.6)$ & $12.2(3.2)$ & 0.76 \\
\hline Physical: & $4.2(1.1)$ & $4.6(1.0)$ & 0.05 \\
\hline Social: & $3.9(1.4)$ & $3.8(1.3)$ & 0.78 \\
\hline Psychological: & $3.9(1.5)$ & $3.8(1.2)$ & 0.62 \\
\hline
\end{tabular}

LCQ, Leicester Cough Questionnaire.

Data expressed as * geometric mean $(95 \%$ confidence intervals), tmedian (interquartile range, IQR) and \$mean (SD). prepubertal children. ${ }^{26}$ Furthermore, an additional enhancement of cough reflex sensitivity occurs in healthy postmenopausal women. ${ }^{17}$ In subjects who have developed a chronic cough, hormonal effects may be exaggerated and could operate via effects on airway inflammatory cells ${ }^{27}$ and/or directly on airway nerves. Sex differences in nociceptive transmission and neuronal sensitisation have been described in experimental pain studies, with evidence suggesting enhanced central sensitisation in women compared with men. ${ }^{28-32}$

It could be argued that the sex differences in cough frequency observed in this study are simply a reflection of the differences in cough reflex sensitivity. To examine this possibility we performed linear regression analyses. Sex and cough reflex sensitivity independently predicted cough rates, suggesting that the degree of cough reflex hypersensitivity is not the sole explanation for the greater rates of coughing in women. It can be clearly seen in fig $4 \mathrm{~B}$ that, overnight (where the main difference in cough frequency was apparent), women had consistently higher cough frequency than men for any given degree of cough reflex sensitivity.

Objective cough rates dramatically fall in a wide range of respiratory conditions overnight. ${ }^{33-35}$ It is generally believed that sleep inhibits coughing, with nocturnal cough occurring predominantly in periods of arousal, but only one study in chronic obstructive pulmonary disease has objectively demonstrated this. ${ }^{36}$ Although it is possible that women with chronic cough experience a greater number of arousals leading to greater nocturnal cough rates, this would be contrary to the objective data in healthy women who have better sleep quality than agematched men. ${ }^{37}$ Perhaps the mechanisms responsible for cough inhibition overnight are less effective in women than in men.

The finding that older subjects with chronic cough had higher cough frequency than younger subjects is counterintuitive. Although it might be expected that respiratory reflexes would deteriorate with age (leading to lower cough rates), previous studies have suggested that cough reflex sensitivity remains stable. $^{38} 39$ However, the relationship between cough frequency and age was independent of the cough reflex sensitivity and also cough duration. We speculate that deficits in inhibitory mechanisms could also explain this phenomenon, similar to the deterioration seen in endogenous inhibitory pain control mechanisms which starts in middle age. ${ }^{40}$

In contrast to previous data generated using the Cough Quality of Life Questionnaire (COLQ), ${ }^{13}{ }^{14}$ we were unable to demonstrate a significantly poorer cough-related quality of life for woman than for men with the LCQ, despite almost double the objective cough frequency. This finding is surprising as, across a wide range of chronic illness, women tend to report worse quality of life than men. While it is possible that women complain less than men about the impact of cough for a given objective cough rate, differences between the items included in the LCQ and COLO may provide a more plausible explanation. On the COLO, women scored more highly in the physical, extreme physical complaints and psychosocial domains. Many of the items describing physical consequences of coughing (eg, retching, vomiting, headaches and dizziness) are not included in the LCO and, in particular, urinary incontinence-which showed the greatest disparity between men and women-is omitted. The LCQ was chosen in this study because, in our experience, patients in the UK had some difficulty with the language used in some of the questions in the COLO. However, these data suggest that the COLO has a distinct advantage over the LCQ in its ability to capture sex differences in chronic cough. 

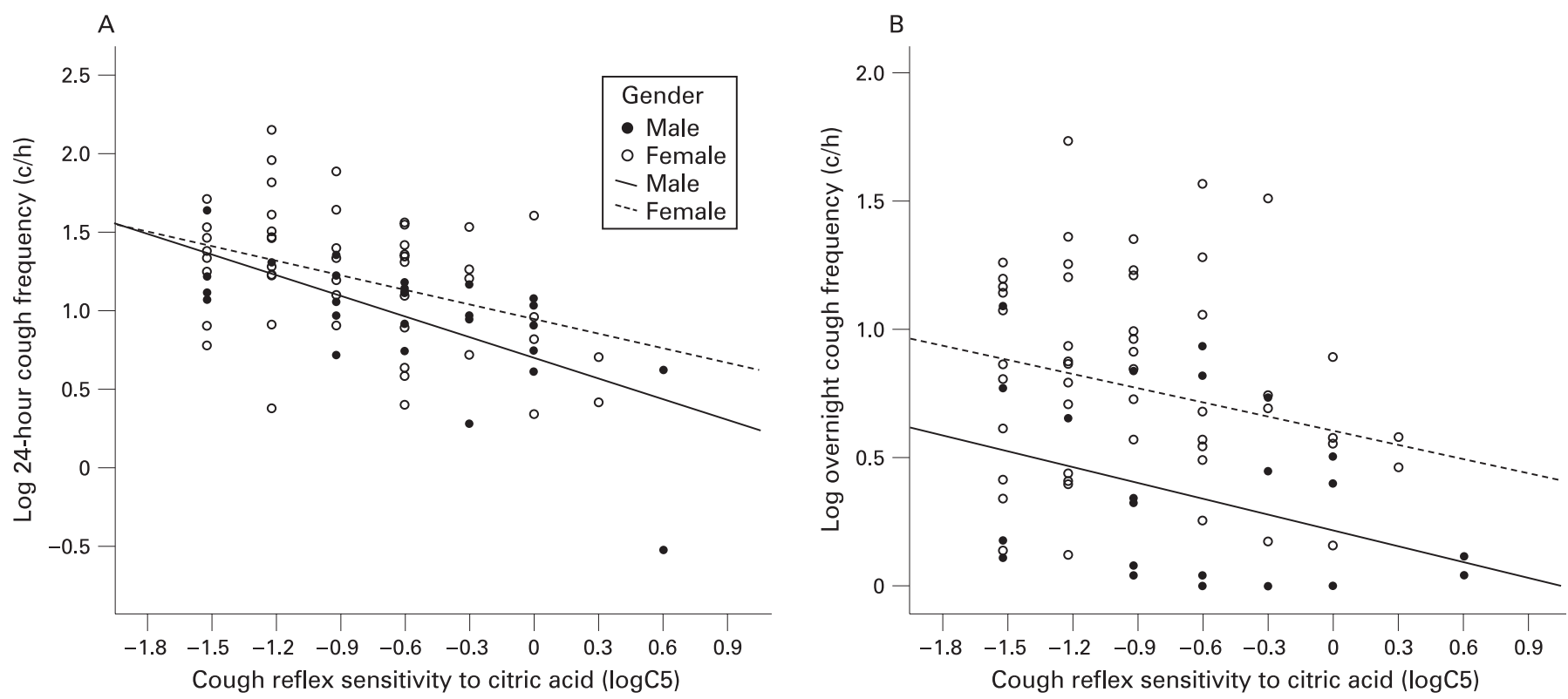

Figure 4 Relationships between cough reflex sensitivity (C5) and cough frequency in men and women (A) over $24 \mathrm{~h}$ and (B) overnight. Regression lines represent the relationships for men and women. Note that over $24 \mathrm{~h}$ the difference in cough rates occurred at higher cough reflex sensitivities whereas overnight women coughed more than men for all levels of cough reflex sensitivity.

Studies in cough will be facilitated by the introduction of automated cough monitors. ${ }^{41}$ Current devices still require laborious manual counting until improved cough detection algorithms are fully validated. In addition, no cough monitor yet allows any measure of cough intensity. Cough intensity may be as important as cough frequency in relating cough to quality of life, and there may be sex differences yet to be explored.

In conclusion, ambulatory objective cough monitoring provides novel insights into the factors modulating chronic cough, forcing us to generate new hypotheses about the mechanisms which may underlie these observations. Furthermore, these findings emphasise that effects of sex and age must be taken into account in the study of cough and when designing clinical trials testing novel antitussive agents.

Funding: This study was funded by a grant from the Moulton Charitable Trust and JAS is funded by a Fellowship from Manchester University. Neither funding body had any involvement in the study design, collecting of the data, analysis or interpretation of the data or in writing of this report.

Competing interests: KM, AW and JAS are inventors on a patent describing novel methods for cough detection and monitoring filed by the University Hospital of South Manchester. KM, AW and JAS have an industrial collaboration and the University Hospital of South Manchester has a license agreement with Vitalograph Ltd to develop a commercial cough monitoring system (Vitalojak).

Ethics approval: Ethics approval was granted by South Manchester local research ethics committee and written informed consent was completed by all subjects.

\section{REFERENCES}

1. Fuller RW, Jackson DM. Physiology and treatment of cough. Thorax 1990;45:425-30.

2. Janson C, Chinn S, Jarvis D, et al. Determinants of cough in young adults participating in the European Community Respiratory Health Survey. Eur Respir J 2001; 18:647-54.

3. Ford AC, Forman D, Moayyedi P, et al. Cough in the community: a cross sectional survey and the relationship to gastrointestinal symptoms. Thorax 2006:61:975-9.

4. Irwin RS, Boulet LP, Cloutier MM, et al. Managing cough as a defense mechanism and as a symptom. A consensus panel report of the American College of Chest Physicians. Chest 1998:114(2 Suppl):133-81S.

5. Haque RA, Usmani OS, Barnes PJ. Chronic idiopathic cough: a discrete clinical entity? Chest 2005:127:1710-3.

6. Irwin RS, Corrao WM, Pratter MR. Chronic persistent cough in the adult: the spectrum and frequency of causes and successful outcome of specific therapy. Am Rev Respir Dis 1981:123:413-7.
7. McGarvey LP, Heaney LG, Lawson JT, et al. Evaluation and outcome of patients with chronic non-productive cough using a comprehensive diagnostic protocol. Thorax 1998;53:738-43.

8. Mello CJ, Irwin RS, Curley FJ. Predictive values of the character, timing, and complications of chronic cough in diagnosing its cause. Arch Intern Med 1996;156:997-1003.

9. Palombini BC, Villanova CA, Araujo E, et al. A pathogenic triad in chronic cough: asthma, postnasal drip syndrome, and gastroesophageal reflux disease. Chest 1999:116:279-84.

10. Poe RH, Harder RV, Israel RH, et al. Chronic persistent cough. Experience in diagnosis and outcome using an anatomic diagnostic protocol. Chest 1989;95:723-8.

11. Simpson G. Investigation and management of persistent dry cough. Thorax 1999;54:469-70.

12. McGarvey LP. Does idiopathic cough exist? Lung 2008;186(Suppl 1):S78-81.

13. French $\mathbf{C T}$, Fletcher KE, Irwin RS. Gender differences in health-related quality of life in patients complaining of chronic cough. Chest 2004;125:482-8.

14. French $\mathbf{C T}$, Fletcher KE, Irwin RS. A comparison of gender differences in healthrelated quality of life in acute and chronic coughers. Chest 2005;127:1991-8.

15. Dicpinigaitis PV, Allusson VR, Baldanti $A$, et al. Ethnic and gender differences in cough reflex sensitivity. Respiration 2001;68:480-2.

16. Prudon B, Birring SS, Vara DD, et al. Cough and glottic-stop reflex sensitivity in health and disease. Chest 2005;127:550-7.

17. Fujimura M, Kasahara K, Kamio Y, et al. Female gender as a determinant of cough threshold to inhaled capsaicin. Eur Respir J 1996;9:1624-6.

18. Fujimura M, Sakamoto S, Kamio Y, et al. Sex difference in the inhaled tartaric acid cough threshold in non-atopic healthy subjects. Thorax 1990;45:633-4.

19. Kastelik JA, Thompson RH, Aziz I, O, et al. Sex-related differences in cough reflex sensitivity in patients with chronic cough. Am J Respir Crit Care Med 2002;166:961-4.

20. Kelsall A, Decalmer S, Webster D, et al. How to quantify coughing: correlations with quality of life in chronic cough. Eur Respir $J$ 2008;32:175-9.

21. Yan K, Salome C, Woolcock AJ. Rapid method for measurement of bronchial responsiveness. Thorax 1983;38:760-5.

22. Birring SS, Prudon B, Carr AJ, et al. Development of a symptom specific health status measure for patients with chronic cough: Leicester Cough Questionnaire (LCO) Thorax 2003;58:339-43.

23. Miller MR, Hankinson J, Brusasco V, et al. Standardisation of spirometry. Eur Respir J 2005;26:319-38.

24. Morice AH, McGarvey L, Pavord I. Recommendations for the management of cough in adults. Thorax 2006;61(Suppl 1):i1-24.

25. Decalmer SC, Webster D, Kelsall AA, et al. Chronic cough: how do cough reflex sensitivity and subjective assessments correlate with objective cough counts during ambulatory monitoring? Thorax 2007;62:329-34.

26. Chang AB, Phelan PD, Sawyer SM, et al. Cough sensitivity in children with asthma recurrent cough, and cystic fibrosis. Arch Dis Child 1997;77:331-4.

27. Mund E, Christensson B, Gronneberg R, et al. Noneosinophilic CD4 lymphocytic airway inflammation in menopausal women with chronic dry cough. Chest 2005; 127:1714-21

28. Fillingim RB, Maixner W, Kincaid $\mathrm{S}$, et al. Sex differences in temporal summation but not sensory-discriminative processing of thermal pain. Pain 1998;75:121-7. 
29. Gazerani P, Andersen OK, Arendt-Nielsen L. A human experimental capsaicin model for trigeminal sensitization. Gender-specific differences. Pain 2005;118:155-63.

30. Riley JL 3rd, Robinson ME, Wise EA, et al. Sex differences in the perception of noxious experimental stimuli: a meta-analysis. Pain 1998;74:181-7.

31. Sarlani E, Grace EG, Reynolds MA, et al. Sex differences in temporal summation of pain and aftersensations following repetitive noxious mechanical stimulation. Pain 2004;109:115-23.

32. Greenspan JD, Craft RM, LeResche L, et al. Studying sex and gender differences in pain and analgesia: a consensus report. Pain 2007;132(Suppl 1):S26-45.

33. Decalmer S, Webster D, Kelsall A, et al. Chronic cough: how do cough reflex sensitivity and subjective assessments correlate with objective cough counts during ambulatory monitoring? Thorax 2007;62:329-34.

34. Smith JA, Owen EC, Jones AM, et al. Objective measurement of cough during pulmonary exacerbations in adults with cystic fibrosis. Thorax 2006;61:425-9.
35. Smith J, Owen E, Earis J, et al. Cough in COPD: correlation of objective monitoring with cough challenge and subjective assessments. Chest 2006;130:379-85.

36. Power JT, Stewart IC, Connaughton JJ, et al. Nocturnal cough in patients with chronic bronchitis and emphysema. Am Rev Respir Dis 1984;130:999-1001.

37. Krishnan V, Collop NA. Gender differences in sleep disorders. Curr Opin Pulm Med 2006;12:383-9.

38. Katsumata U, Sekizawa K, Ebihara T, et al. Aging effects on cough reflex. Chest 1995; 107:290-1.

39. Chang AB, Widdicombe JG. Cough throughout life: children, adults and the senile. Pulm Pharmacol Ther 2007:20:371-82.

40. Lariviere $\mathbf{M}$, Goffaux $P$, Marchand $\mathbf{S}$, et al. Changes in pain perception and descending inhibitory controls start at middle age in healthy adults. Clin J Pain 2007:23:506-10.

41. Smith J. Ambulatory methods for recording cough. Pulm Pharmacol Ther 2007;20:313-8

\section{Pulmonary puzzle}

\section{Bilateral pneumothoraces, cystic lung disease and papular skin lesions in a young man}

\section{CLINICAL PRESENTATION}

A 36-year-old non-smoking Korean man presented to the emergency room with bilateral pleuritic chest pain and mild dyspnoea on exertion. His review of systems was negative. He had multiple family members across three generations with "lung disease". His physical examination was notable for a normal body habitus, left greater than right diminished breath sounds, hypertympany of the left thorax and many small (2$3 \mathrm{~mm}$ ) papular lesions on his neck, chest and upper back (fig 1A). These had been present since about the age of 30 years and over the last 6 years the number of lesions had steadily increased with some larger lesions on his upper back. An initial chest radiograph showed a small right and a large left pneumothorax. In addition to the pneumothoraces, a CT scan demonstrated multiple bilateral, basilar and peripheral predominant thin-walled cystic parenchymal lesions (fig 1B).

Bilateral chest tubes were placed with resolution of the right pneumothorax. Owing to a persistent left-sided air leak, the patient underwent video-assisted thoracoscopic surgery with resection of multiple cystic lesions and a mechanical pleurodesis. Lung pathological examination demonstrated benign intraparenchymal thin-walled cysts which were negative for organisms and immunohistochemical stains (CD1a, HMB-45, S-100 and smooth muscle actin).

\section{QUESTIONS}

What is the diagnosis and what diagnostic test confirmed this diagnosis?

See page 457

This case was submitted by:

\section{A R Haas, ${ }^{1}$ K L Nathanson ${ }^{2}$}

${ }^{1}$ Clinical Operations, Section of Interventional Pulmonology and Thoracic Oncology, University of Pennsylvania Medical Center, Philadelphia, Pennsylvania, USA; ${ }^{2}$ Division of Medical Genetics, Department of Medicine, University of Pennsylvania School of Medicine, Philadelphia, Pennsylvania, USA

Correspondence to: Dr A R Haas, Clinical Operations, Section of Interventional Pulmonology and Thoracic Oncology, University of Pennsylvania Medical Center, 823 West Gates Building, 3400 Spruce Street, Philadelphia, PA 19104, USA; arhaas@ uphs.upenn.edu

Competing interests: None.

Patient consent: Obtained.

Thorax 2009;64:398. doi:10.1136/thx.2008.102095
Figure 1 (A) Papular lesions on the upper back and neck. (B) CT scan of the chest showing bilateral pneumothoraces and basilar and peripheral predominant thin-walled cystic lesions.
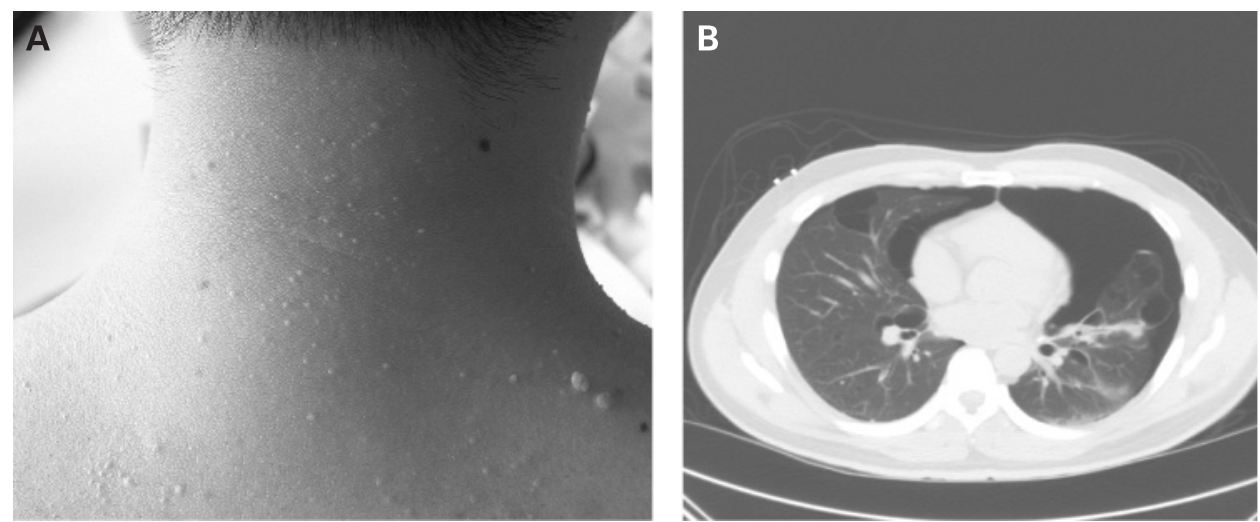